\title{
Die Lappendammbildung bei completer Ruptur, besonders die Lawson Tait'sche Methode ${ }^{1}$ ).
}

\author{
Von \\ Wilhelm Nagel, \\ Berlin. \\ (Mit 6 Figuren ind Text)
}

Es liegt nicht in meiner Absicht, die ganze Frage der Dammplastik durch Lappenbildung mit ihren zahlreichen Modificationen wieder aufzurollen, noch beabsichtige ich statistisches Material ${ }^{2}$ ) vorzubringen. Es sind vielmehr nur einige technische Punkte in der sogenannten Lawson Tait'schen Methode, welchen ich näher treten möchte, und die, soweit ich die Literatur übersehe, lange nicht nach Gebühr berücksichtigt werden. Dass die Tait'sche Dammplastik von Anfang an so zahlreiche Modificationen erlitten hat, ja dass sie heute noch, nach den Operationsberichten zu schliessen, eigentlich gar nicht richtig ausgeführt wird, beruht gerade, meiner Ansicht nach, auf der äber die gedachten technischen Fragen herrschenden Unklarheit. Tait selbst wird vielfach die Schuld hieran beigemessen, weil er in dem ersten Vortrage über seine Methode (Transact. of the Obst. Society in London, 1879) es unterliess, das Princip derselben gebührend hervorzuheben. In der That erklärte in der auf seinen Vortrag folgenden Discussion der eine Redner nach dem andern, dass das Prineip und der Gang der Operation ihm vollkommen unklar geblieben. Durch die Lectüre des betreffenden Artikels und Betrachtung der beigegebenen ersten Vortrag.

1) Nach einem in der geburtshülll. Gesellschaft zu Berlin gehaltenen

2) Dasselbe ist yon M. Besser in seiner Dissertation: Die Erfolge der Lawson-Tait'schen Dammplastik bei completen Dammrupturen, Berlin 1902 bearbeitet worden. 
Figur gelangt man auch nicht zu einem besseren Verständniss. Man darf aber nicht vergessen, dass Lawson Tait ursprünglich gar nicht die Priorität dieser Operation für sich in Anspruch nahm; er führte sie wohl so aus, wie er es bei seinen Lehrern gesehen hatte und fühlte sich deshalb nicht berufen, mit dem Princip derselben sich besonders zu beschäftigen. Bekanntlich ist Lawson Tait's Name zuerst durch seinen Schüler Heiberg mit der Operation in Verbindung gebracht worden.

- Später versuchte man ältere Namen mit der Operation zu verknüpfen. Sänger schreibt ihre Entdeckung Voss (Christiania, 1865), Berry Hart und Barbour dem John Duncan, G. E. Herman dem Jonathan Hutehinson zu. In Wirklichkeit dürfte die Operation viel älter sein. Hier in Deutschland hatte, wie Olshausen in der auf Sänger's Vortrag folgenden Discussion betonte, v. Langenbeck (1853) eine Lappenspaltungs-Methode, Perineo-synthesis, durch Biefel beschrieben. Alle diese Methoden weichen indessen nicht unwesentlich von einander ab sowohl in der Sehnittführung, wie in der Naht.

Die Tait'sche Dammplastik ist in anatomischer Beziehung so logisch und das Princip derselben so wohl durchdacht, dass es wahrlich der Mühe werth ist, mit demselben sich vertraut zu machen. Ich habe bei verschiedenen Gelegenheiten, zuletzt auf dem internationalen Congresse in Madrid, versucht, der gedachten Operation in ihrer ursprünglichen Gestalt gerecht zu werden. Es will mir aber scheinen, als sei der Werth derselben noch nicht gebührend anerkannt, und deshalb gestatte ich mir, noch einmal auf sie einzugehen.

Ich bemerke noch, dass meine Auseinandersetzungen nur auf die Operation eines vernarbten completen Dammrisses sich beziehen. Auf die Lappenbildungsmethode zur Heilung eines incompleten Dammrisses, oder gar eines Scheidenvorfalles lasse ich mich in keiner Weise ein.

Weil er, wie gesagt, Anfangs das Princip der Operation sich nicht klar gemacht hatte, änderte Lawson Tait öfters sein Verfahren. So kannte Meinert ${ }^{1}$ ) vier Methoden, nach welchen Tait alte Dammrisse operirte. In seinem später erschienenen Lehrbuch giebt Tait ${ }^{2}$ ) als Princip seiner Methode an, mittels Lappen-

1) Centralbl. f. Gyn. 1888. No. 40.

2) Diseases of women and abdominal surgery. eLeicester. 1889. 
spaltung don Riss in seiner ursprünglichen Gestalt herzustellen und als A ufgabe der Naht, die Wunde rechtwinklig zum transversalen Schnitt, das heisst also, in der Richtung der Raphe perinei zu vereinigen. Diese Definition - "quer schneiden und längs vereinigen", wie Küstner ${ }^{1}$ ) sich ausdrückt - findet man in allen Wiedergaben der Tait'schen Operation. Sie trifft aber meines Erachtens nicht die Hauptsache, und Niemand ist im Stande, hiernach die Operation richtig auszuführen. Daher auch der häufige, aber ungerechte Vorwurf gegen dieselbe, dass Theile auf einander genäht werden, die gar nicht zusammengehören.

Die beste Definition der Aufgabe der Lappen-Perineorraphie giebt entschieden Sänger 2): "Durch Spaltung des in einen Narbensaum auslaufenden Septum recto-vaginale wird die evertirte Scheide auf ihr früheres Niveau gehoben, die vordere Mastdarmwand wieder hergestellt. Von den Seiten her schiebt sich zwischen Soheide und Mastdarm der durch den Riss entzweite Damm wieder ein, wie wenn er nie verletzt worden wäre, und so genau, dass die durchrissenen und nach aussen vorgezogenen Enden und Fascien den Musculi constrictor cunni und transversus perinei in derselben Lage wieder zusammengefügt werden, welche sie vor der Durchreissung eingenommen haben." Diese Definition lässt an Klarbeit nichts zu wünschen übrig. Sänger's $\left.{ }^{3}\right)$ Darstellung von der Leistung der Operation ist aber nicht ganz richtig, weil Scbeide und Rectum in derselben eine unnatürliche Lage einnehmen, gewissermaassen ein Dach über dem nengebildeten Perineum bilden.

Ferner entsprach die Art, in welcher er die Wunde zusammennähte, nicht den natürlichen Verhältnissen, und so erklärt es sich, warum Sänger bei. seinen verdienstrollen Bestrebungen, die LappenDammoperation in Deutschland einzuführen, auf so grossen Widerstand stiess ${ }^{4}$ ) and fast jeder Operateur die Methode modificirte.

Ich führe einige dieser Modificationen an. So sagt Fritsch5): Es wird zunächst durch zwei Schnitte beiderseitig der Mastdarm von der Scheide abgetrennt. Man dringt ungefähr $1 \frac{1}{2} \mathrm{em}$ tief

1) Ueber Episioplastik. Samml, klin. Vorträge. Neue Folge 16 (42).

2) Verh. d. deutschen Gesellsch. f. Gyn. Freihurg. 1889.

3) Sammlung klin. Vorträge, herausgegeben von R. Volkmano. No. 301.

4) Siehe die auf Zweifel's und Sänger's Vorträge folgenden Diskussionen auf dem Congresse deatscher Gynälrologen Halle 1888 and Freiburg 1889.

5) Centralbl. f. Gyn. 1887. No. 30. 
ein, so dass beide Organe völlig unabhängig von einander beweglich sind. Verlaufen die Schnitte gerade auf der Höhe des scharfen Grates der Narbe, so wird der Wundrand oft etwas unregelmässig, dann wird er durch Scheerenschnitte gerade gemacht. Hierauf löst man die zwei Sphincterenden, welche sich fast immer durch eine kleine trichterartige Einziehung am Afterrande markiren, ab, und führt eine Nadel provisorisch durch die beiden Enden. Zieht man den nicht geknüpften Faden stark abwärts, so stellt man die natürliche Form der Mastdarmverhältnisse wieder her. Es klappt sich dabei meist die evertirte Mastdarmschleimhaut so zurück, dass die frischen Wundflächen an einander liegen. Fasst man nun gleichzeitig den oberen vaginalen Wundwinkel an und zieht ihn nach oben, so erscheinen zwei Paar Wundränder, in der Tiefe die kürzere, meist nur 2 bis höchstens $4 \mathrm{~cm}$ lange Mastdarmwunde und die längere, 7-8 $\mathrm{cm}$ lange Vaginalwunde darüber. Nunmehr wird der Mastdarm zuerst von der Wunde aus genäht; dann folgt die Naht der Scheide, schliesslich diejenige des Dammes. "

A. R. Simpson zertrent den Scheidenlappen durch einen Medianschnitt in zwei Lappen. Sein Verfahren ist so: Längsschnitt durch die Mitte der Columna bis auf die Haut des Dammes. Dann beiderseitiger, stark gekrümmter Bogenschnitt bis zur Uebergangsstelle der kleinen in die grossen Labien. Abpräpariren der beiden Lappen, welche man lateralwärts möglichst dick zu machon sucht. Emporheben derselben gegen die Scheide hin und unter ihnen Schluss der gesammten Wundhöhle durch 1-2 Etagen versenkter Catgutnaht und oberfläehlicher Drahtsuturen.

Einige andere Modificationen, wie die von Stratz und von von Grunwaldt1), welche den Vaginallappen nach oben spitz präparirten, haben keine Beachtung gefunden.

In der den Vorträgen von A. Martin ${ }^{2}$ ) und Sänger sich anschliessenden Diskussion ${ }^{3}$ ) wurden neue Gesichtspunkte nicht vorgebracht; sowohl complete wie incomplete Rupturen wurden als für die Operation geeignet empfohlen, obwohl der complete Riss als eigentliche Domäne der Lappenplastik anerkannt wurde. Die Frage, ob Catgut oder unresorbirbares Material verwendet werden

1) Centralbl. f. Gyn. 1890.

2) Berliner klin. Wochenschr. 1889. No. 6.

3) Verh. d. geburtsh. Gesellsch. zu Berlin, Sitzung vom 11. Januar und 8. Februar 1889. 
soll, nahm einen breiten Raum in der Diskussion ein. Das Grundprincip der Methode wurde aber nicht berührt; nur geht aus Bemerkungen von Sänger und Martin hervor, dass die Nähte aussen auf dem Damm zu liegen kommen.

Küstner ${ }^{1}$ ) verwirft eine schematische Schnittührung und will, dass die Schnitte entsprechend der Narbe gelegt werden müssen, bald schief, schräg und mehr weniger auf einer Seite.

Als Beispiel, wie wenig klar das Princip der Tait'schen Operation geblieben war, mag tolgender, am Schlusse der über mehrere Jahre sich erstreckenden Diskussion geschriebener Satz von v. Grunwaldt ${ }^{2}$ ) gelten: "Der durch Lappenspaltung gewonnene grosse rechteckige Lappen findet keine befriedigende Verwendung und muss zum Theil nekrotisch zu Grunde gehen." Eine ähnliche Ansicht batte übrigens auch früher $\operatorname{Son} n \operatorname{tag}^{3}$ ) bereits ausgesprochen.

Um nun entscheiden zu konnen, ob es überhaupt möglich sei, die Wunde in ihrer ursprüngliohen Gestalt wieder herzustellen und den richtigen Weg hierzu festzulegen, müssen wir auf das Ausschen eines frischen completen Darmrisses und die Art seiner Vernarbung, wenn nicht genäbt, zurückgreifen.

Ein frischer completer Dammriss hat bekanntlich die in Fig. 1 wiedergegebene Gestalt. Spannt man durch Zug an beiden Seiten die Wunde, so nimmt sie eine rautenförmige Gestalt (Fig. 2) an. Ein sich selbst überlassener completer Dammriss verheilt in der Weise, dass Vaginal- und Rectalrand mit einander verwachsen, weil, wie Küstnerit) sich ausdrückt, die sugillirte, ödematöse Columna posterior diek und schwer sich in die Wunde hineinschiebt, während die seitlichen Flächen des entzweigerissenen Dammes nach beiden Seiten ausweichen, um dort für sich zu vernarben. Gleichzeitig wird, wohl durch den Zug der sich retrahirenden seitlichen Partien, die recto-vaginale Brücke hervorgezogen, so dass sie au niveau mit der umgebenden Haut liegt. In dem Narbengewebe za beiden Seiten der Brücke bemerkt man häufig genug jederseits eine Delle, hervorgerufen durch den durchrissenen und schrumpfenden Sphincter ani.

Will man nun die ursprünglichen Wundverhältnisse durch Lappenspaltung wieder herstellen, so muss der Hautschnitt die

1) Congress d. deutsch. Gesellsoh.f. Gyn. Freiburg 1889.

2) Centralbi. f. Gyn. 1890. No. 31.

3) Verh. d. deutsch. Gesellsoh. f. Gyn. Freiburg 1889.

4) $1, \mathrm{c}$. 
Figur 1.

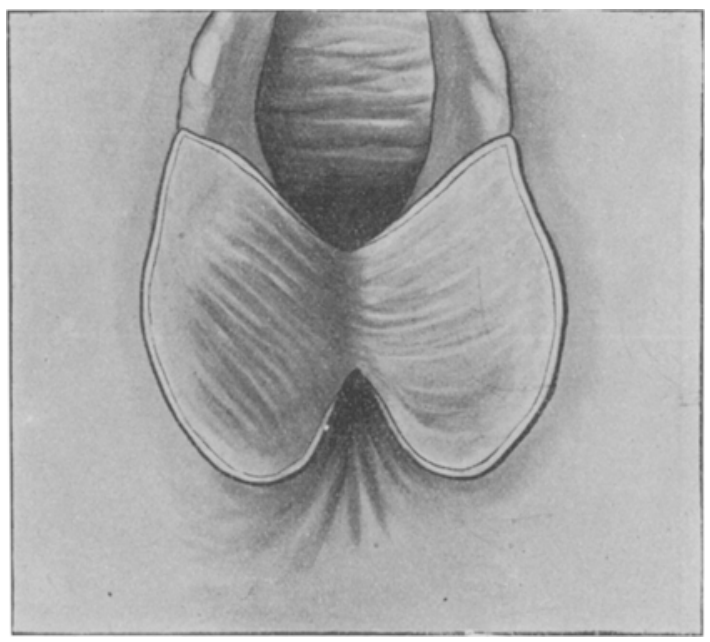

Figur 2.

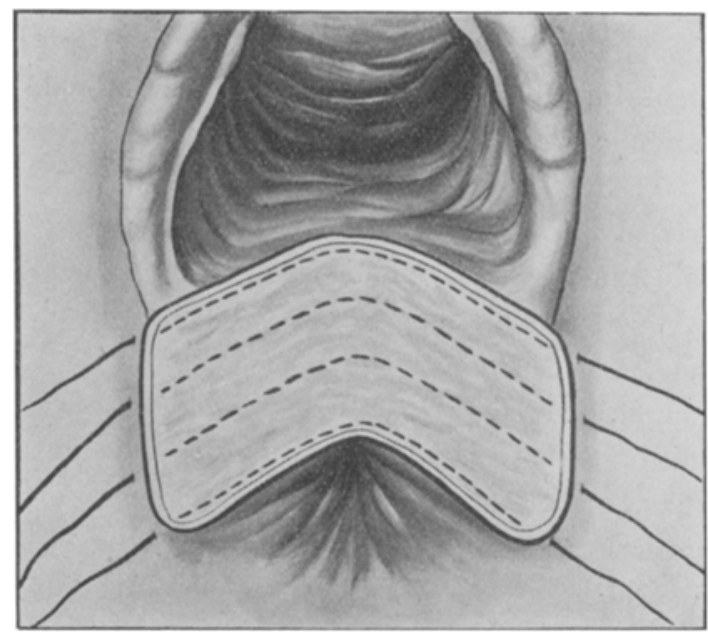

von dem Erfincier angegebene Form, nämlich ein lateinisches $H$ mit nach aussen concaven Schenkeln zeigen (Fig. 3). Die einfache Trennung von Scheide und Mastdarm genügt keinesfalls bei completer Ruptur. Die von Schatzi) ausgesprochene Befürchtung,

1) Congress der Gesellschaft deutscher Gynaekologen. Freiburg. 1889. 
Figur 3

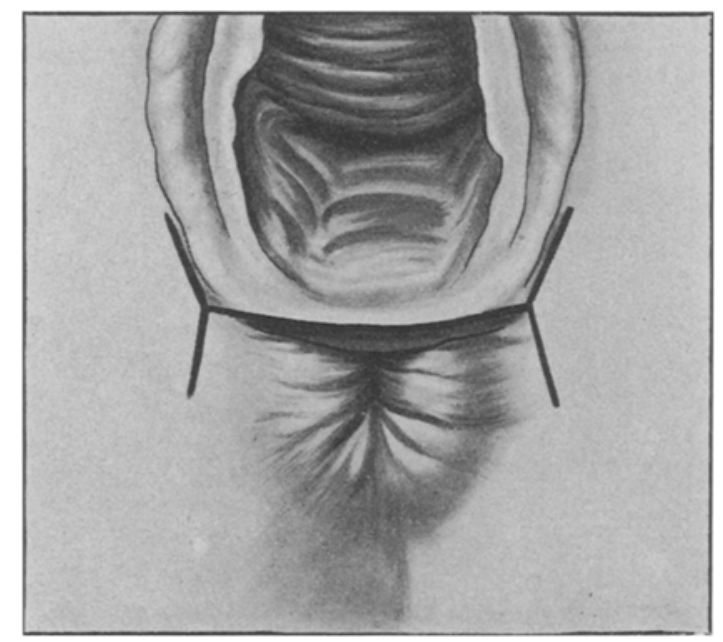

dass hierdurch der Scheideneingang zu weit wird, kann sich wohl nur auf die Fälle beziehen, in welchen wegen Prolaps, womöglich in Folge Erschlaffung des Beckenbodens operirt wird, Fälle, die aber gar nicht für die Lawson-Tait'sche Methode sich eignen.

Die Aufgabe des horizontalen Schenkels des Schnittes ist, Mastdarm und Scheide wieder von einander zu trennen, er darf also nur soweit zu beiden Seiten reichen wie die Scheide. Ich gebe deshalb auch stets meinen Schülern den Rath, in Gedanken zwei Scheidentangenten zu ziehen; dort wo dieselben das seitliche Narbengewobe schneiden, liegen die Endpunkte des Horizontalsehnittes. Hieraus ergiebt sich aber, dass dic vier seitlichen Schnitte, entsprechend der Rundung des Introitus vaginae und des Anus, nach aussen divergiren müssen. Nacht man sie nämlich senkrecht, wie in verschiedenen Modificationen des Tait'schen Verfahrens, z. B. in der von $\mathrm{Pozzi}^{1}$ ) angegebenen, so gehen sie, bei sonst richtiger Abmessung der Länge des Horizontalschnittes, in die Scheide hinein. Will man hinwiederum unter Beibehaltung der senkrechten Schnitte die Berührung der Scheide vermeiden, so muss man den Horizontalsehnitt nach beiden Seiten entsprechend verlängern. In beiden Fällen schafft man sich ganz unnatürliche Wundverhältnisse: gehen die Schnitte in die Scheide

1) Traité dẹ Gynécologie. Paris. $189 \%$. 
hinein, so zerstört man den oberen vaginalen Wundrand, macht man den Horizontalsehnitt zu lang, so gewinnt die Wunde eine unverhältnissmässige Ausdehnung und die seitliche Lappenspaltung findet womöglich ganz ausserhalb der Narbe statt, so dass nun Theile auf einander genäht werden, die nicht zum Damme gehören. Die Folgen sind: nachträgliche Spannung im Bereich der Wunde, über welche die Operirten, von $O t^{1}{ }^{1}$ ) zufolge, häufig klagen und eine allmählich entstehende Vertiefung der Narbe am Damm.

Die Länge der beiden seitlichen Schnitte richtet sich im Uebrigen nach der Ausdehnung des dort belegenen Narbengewebes. Mastdarm und Scheide dürfen nicht unnöthig weit getrennt werden. Durch eine za weit ausgedehnte Trennung der beiden Röhren werden die beiden Lappen ausserdem zu dünn, in Folge dessen die Gefahr des Brandigwerdens und der Durchlochung derselben näher rückt. Die Lappen werden vielmehr vorwiegend durch ausgiebige Vertiefung der seitlichen Schnitte gebildet; dadurch werden die Lappen auch an ihrer Basis gehörig dick und somit, wie Sänger betont, in ihrer Ernährung gesichert.

Ist nun die Lappenbildung beendet, müssen die vier Ecken mit je einer feinen Hakenzange gefasst werden und dor obere Lappen nach oben, der untere nach unten geschlagen. Etwa in der Mitte der Wunde sich spannendes Gewebe wird mit der Scheere durchschnitten, bis die Wunde eine rautenförmige Gestalt bekommen hat. Nun entspricht sie vollkommen der Gestalt eines hervorgezogenen frischen Dammrisses: der obere Rand der soeben geschaffenen Wunde entspricht dem Vaginalrand, der untere Rand dem Rectalrand, die Seitenränder den Hauträndern des frischen Dammrisses.

Sänger²) lehrte anders, indem er zeigte, dass man die Mitte der Lappen in eine Arterienpincette fassen soll und damit den oberen Lappen nach oben, den unteren nach unten ziehen "wodurch die ganze Wunde in eine vertikale Eibene kommt." (Fig. 4.)

Diese Lehre Sänger's ist wohl hier zu Lande überall massgebend gewesen, sie ist aber nicht richtig und hat wesentlich den vielfachen Widerstand gegen die Operation und die zahlreichen Modificationen derselben verschuldet. Fasst man nämlich die Lappen, wie Sänger, so erhält die Wunde, wie wir gleich sehen

1) Dieses Archiv. Bd. 39.

2) Samml. klin. Vorträge. No. 301. 
Figur 4.

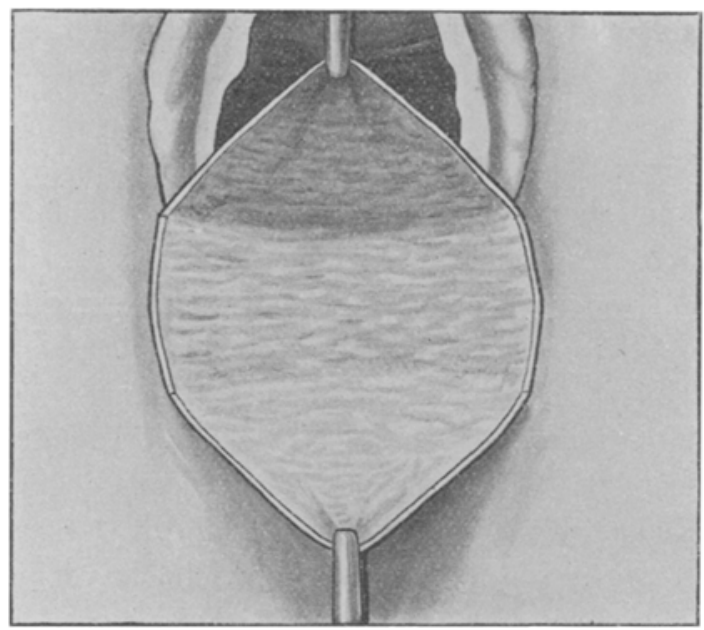

werden, eine unnatürliche und lür dic $\mathrm{Naht}$ ganz ungünstige Gestalt (Fig. 4).

Betrachten wir jedoch zuerst die für die Naht gegebenen Vorschriften:

Lawson Tait beschreibt die Naht folgendermaassen: die oberste Naht wird zuerst angelegt. Die Nadel kann von jeder Seite her in das Septum oingefübrt werden, etwa ein Zoll ron der Spitze der Wunde and die Ein- und Ausstichöffnung muss stets an der Umschlagestelle des Tappens liegen. Die Nadel wird etwa 1/4 Zoll von der Spitze der Wunde ausgestochen, wieder eingestochen, um schliesslich auf einer der Einstichöfnung entsprechenden Stelle herauszukommen. Die hintere Ligatur wird gerade vor dem zurückgeschlagenen Lappen angelegt.

Auf der in Tait's später ersehienenem Lehrbuche wiedergegebenen Figur liegen sümmtliche Nähte horizontal, parallel dem oberen und unteren Rand der quer rechteckigen Wunde; die Einund Ausstichsöfnnungen befinden sich statt auf der äusseren Haut in der Wunde selbst, etwa 2-3 $\mathrm{mm}$ rom Wundrande entfernt.

Während eines Besuches in Birmingham (1892) batte ich durch das liebenswürdige Entgegenkommen Tait's Gelegenheit, bei einer Dammplastik wegen completer Ruptur zu assistiren. Nach Bildung der Lappen in oben beschriebener Weise wurden dieselben mittels je zwei Klemmpincetten nach oben und unten geschlagen 
und sämmtliche vier Nähte quer angelegt, ohne der ersten und vierten Naht besondere Aufmerksamkeit zu widmen. Beim Knoten der Nähte wurden die Pincetten einwärts gerollt, wodurch die Entstehung der Pürschel (s. unten) vermieden werden sollte.

Sänger legt ebenfalls sämmtliche Nähte quer an ohne besondere Vorschriften für die obere und untere Randnaht, so dass sowohl oberer wie unterer Lappen für sich vernäht wird, wodurch die Entstehung eines Pürschels unvermeidlich wird. Bei Knotung der Naht beobachtete Sänger, dass der Rand des Scheidenlappens öfters in eine nach vorn offene Falte oder in eine gekräuselte kleine Rosette sich legte. Diese Rosette lernte er immer kleiner zu machen, so dass er in ciner ganzen Reihe von Fällen gar keine Nekrose derselben mehr beobachtete. Dieses erreichte er theils durch Herstellung dicker Lappen, theils dadurch, dass er durch den oberen queren Rand des Scheidenlappens einen etwas feineren Silberdraht legte. Hierbei ist darauf zu achten, dass der bier sehr dünne Lappen nicht durchstochen, sondern dass die Nadel immer unter der Schleimhautoberflärhe geführt werde: beim Zusammenziehen des Silberdrahtes verschwindet der Rand des Scheidenlappens vollständig oder mit Hinterlassung eines feinen Spaltes, den man allenfalls noch durch eine oberflächliche Naht schliessen kann.

Zweifel 1) folgt, ebenso wie Sänger, der Methode Tait's noch am genauesten, aber seine Anweisung zur Naht, welche er "mehr schräg führt in der Achse der Scheide, um dadurch grössere Flüchen in Berührung zu bringen", ist nicht ganz klar. Für die Vereinigung des Seheiden-Mastdarmspaltes braucht Z weifel in der Regel nur 2, höchstens 3 Scheidennähte; zur genauen Vereinigung des Dammes kommen noch nach Bedarf 3-4 Dammnähte hinzu.

In diesen, wie auch in den übrigen Anweisungen zur Naht, die ich deshalb hier nicht anführe, liess man die topographischen Verhältnisse ausser Betracht und legte, wie man sieht, nur Gewicht auf die quere Einführung der Nähte. Dadurch erhielt man aber ein ganz unbefriedigendes Resultat, indem, wie bereits gesagt, vorn und hinten der Pürschel entstand, wolcher auf dem Congress in Freiburg die Kritik Hegar's

1) Ueber Perineoplastik. Verh. d. deutschen Gesellsch. f. Gyn. Halle, 1888. S. 97 . 
herausforderte. Die Folge waren zahlreiche Modjficationen der Naht, von denen aber keine einzige das richtige traf.

Man braucht nur einen frischen completen Dammriss zum Vergleich heranzuziehen, um sich sagen zu können, dass die oben erwähnte, allgemein übliche Naht falsch ist. Es fällt doch Niemandem ein, einen frischen completen Dammriss in der Weise, wie z. B. bei der Sänger'schen Figur (siehe Fig. 4) in die Länge zu zerren und so zu nähen! Der frische complete Dammriss wird doch entweder in der Weise vereinigt, dass Rectum, Scheide und Damm der Reihe nach für sich genäht wird, oder so, dass von der äusseren Haut her Nähte parallel dem Vaginal- und Rectalrande angelegt werden (Figur 2) (die sogenannte Emmetsche Naht).

Die erstgenannte (klassische) Methode ist, dank der durch die Vernarbung entstandenen Herrorzerrung ron Scheide und Mastdarm, bei der Lappendammbildung für gewöhnlich nicht anwendbar, sondern nur in denjenigen Fällen, in welchen der Riss sich sehr hoch hinauf erstreckt, oder die Operation verhältnissmässig früh ausgeführt wird, ehe die Narbenverzerrung ihre volle Wirkung ausgeübt hat. In solchen Fällen sieht man nämlich den oberen und unteren Rand der new hergestellten Wunde keine gestreckte, sondern eine gebrochene Linie, nämlich ein die Spitze nach innen kehrendes $\vee$ bilden. Bei zurückgeschlagenen Lappen kehren selbstredend die beiden $V$ ihre Spitzen gegen einander $\left(\begin{array}{l}V \\ N\end{array}\right)$. Hier ist es denn in der That mög. lich, die neu hergestellte Wunde in der klassischen Weise zu behandeln, indem Rectum und Seheide für sich und zuletzt der Damm vernäht wird.

Cullingworth ${ }^{1}$ ) wendet stets diese Nahtmethode an, wohl ein Zeichen, dass ungeheilte complete Risse von grosser Ausdehnung ihm verhältnissmässig früh zur Operation zugewiesen werden.

In den meisten Fällen aber lässt die neugeschaffene Wunde nur nach der von Emmet bei frischen Rissen angewandten Methode (Fig. 2) sich vernähen und das Princip dieser hat offenbar dem Erfinder der Lappendammbildung, wem es auch sei, vorgeschwebt, als er die Anweisung gab, die Wunde "querts zu vernähen.

Die Emmet'sche Naht eines frischen completen

1) St. Thomas Hospital Reports. Bd. 27. 
Dammrisses wird bekanntlich in der Weise ausgeführt, dass die oberste Ligatur den Vaginalrand, die unterste den Rectalrand entlang geführt wird, während zwei mittlere Ligaturen das dazwischen liegende Gewebe fassen; sämmtliche Ein- und Ausstichöffnungen liegen auf der äusseren Haut. Wendet man nun dieses Princjp auf die Vernähung der durch Lappenspaltung erzeugten Wunde an, so ist es ganz klar, dass Ein- und Ausstich der oberen Jigatur dicht unter dem von Vaginallappen und äusserer Haut gebildeten Winkel, Ein- und Ausstich der untersten Ligatur dicht über dem von Rectallappen und ïusserer Haut gebildeten Winkel liegen müssen, während in beiden Fällen der Faden genau den oberen und unteren Rand entlang geführt werden muss (Fig. 5). Die beiden mittleren Ligaturen werden einfach quer durch die Wunde geführt.

Figur 5.

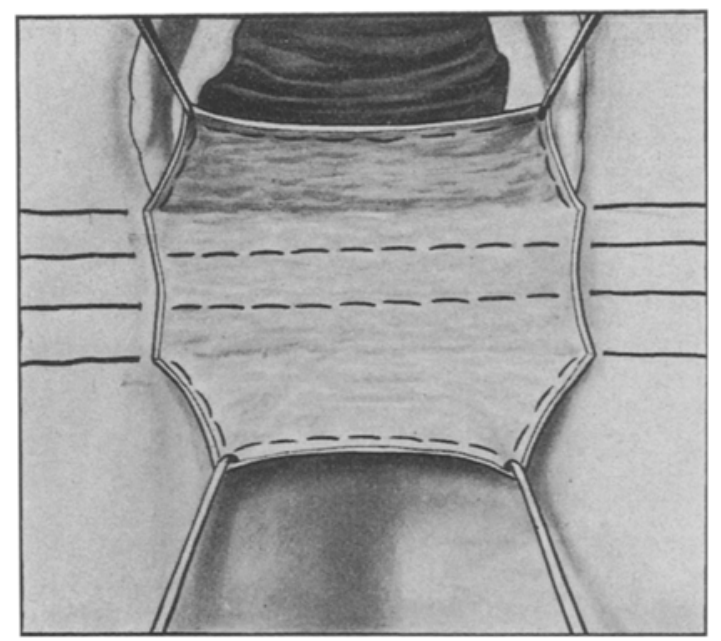

Sobald aber die Einstiche und Ausstiche über dem oberen, beziehungsweise unter dem unteren der bezeichneten Winkel liegen, werden Rectal- und Vaginallappen für sich vernäht, so dass, ähnlich wie bei den übrigen Methoden, vorn und hinten der berüchtigte Pürschel entsteht.

Bei keinem der Autoren, die sich mit der Dammplastik durch Lappenbildung beschäftigen, habe ich eine Andeutung von dieser Art der Anlegung der oberen und unteren Naht gefunden und doch ist darin der Cardinalpunkt der Methode zu suchen. Nur in der 
Dissertation eines jungen russischen Arztes (Rodsewitsch, Schüler Slaviansky's) aus dem Jahre 1889 ist angegeben, dass längs dem freien Rande des losgetrennten Lappens ein Faden durehzuziehen sei, wodurch der Lappen nach Art eines Tabaksbeutels zusammengeschnürt und zum. Schrumpfon gebracht wird. Bis jetat ist es mir nicht gelungen, das Original zu Gesicht zu bekommen, vielleicht hat aber Rodsewitsch das Richtige im Sinne gehabt und die ihm von v. Ott ${ }^{1}$ zu Theil gewordene Kritik ist demnach ganz unberechtigt. V. Ott wendet sich besonders deshalb, gegen die Tabaksbeutelnaht, weil dureh dieselbe Rosetten rorn und hinten entstehen sollen, die er als entstellende Kunstproducte erk]ärt. Dem sei, wie ihm wolle, durch die von mir hier angegebene Nahtmethode entstehen jedenfalls keine Rosetten. (Fig. 6.)

Figur 6.

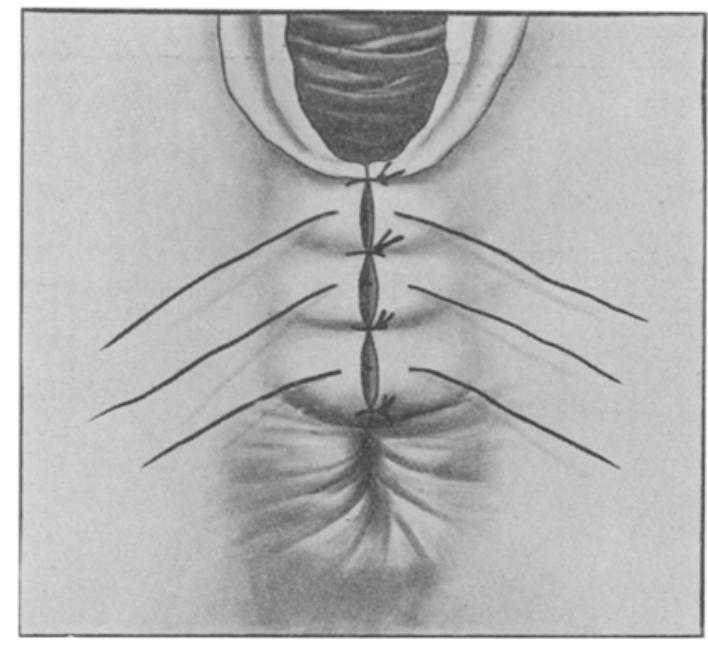

Wie man sieht, besteht also das Prineip der sogenannton Tait'schen Methode darin, durch Lappenspaltung eine Wunde zu schaffen, die topographisch mit einem frischen completen Dammriss übereinstimmt und die nach Emmet vernäht wird. Der Erfinder dieser wohl durchdachten Methode ist unbekannt und wohl ausserhalb der Reihe der von den Autoren angeführten za suchen. Jedenfalls muss man ihm die Anerkennung eines genialen Praktikers zu Theil werden lassen.

1) Dieses Archiv. Bd. 39. 